\title{
Review Article \\ Splicing Regulation: A Molecular Device to Enhance Cancer Cell Adaptation
}

\author{
Vittoria Pagliarini, ${ }^{1,2}$ Chiara Naro, ${ }^{1,2}$ and Claudio Sette ${ }^{1,2}$ \\ ${ }^{1}$ Department of Biomedicine and Prevention, University of Rome Tor Vergata, 00133 Rome, Italy \\ ${ }^{2}$ Laboratory of Neuroembryology, Fondazione Santa Lucia, 00143 Rome, Italy \\ Correspondence should be addressed to Claudio Sette; claudio.sette@uniroma2.it
}

Received 30 January 2015; Accepted 23 March 2015

Academic Editor: Peter Jordan

Copyright (c) 2015 Vittoria Pagliarini et al. This is an open access article distributed under the Creative Commons Attribution License, which permits unrestricted use, distribution, and reproduction in any medium, provided the original work is properly cited.

\begin{abstract}
Alternative splicing (AS) represents a major resource for eukaryotic cells to expand the coding potential of their genomes and to finely regulate gene expression in response to both intra- and extracellular cues. Cancer cells exploit the flexible nature of the mechanisms controlling AS in order to increase the functional diversity of their proteome. By altering the balance of splice isoforms encoded by human genes or by promoting the expression of aberrant oncogenic splice variants, cancer cells enhance their ability to adapt to the adverse growth conditions of the tumoral microenvironment. Herein, we will review the most relevant cancer-related splicing events and the underlying regulatory mechanisms allowing tumour cells to rapidly adapt to the harsh conditions they may face during the occurrence and development of cancer.
\end{abstract}

\section{Introduction}

The transcription units of most eukaryotic genes are characterized by exons, short regions of approximately $200 \mathrm{bp}$ containing untranslated and coding sequences, interspersed between large noncoding introns (generally $\geq 1000 \mathrm{bp}$ ) [1]. Removal of intronic sequences and joining of exons is one of the key events in the multistep process ensuring maturation of pre-mRNAs into mRNAs [2]. This process, called splicing, is carried out by the spliceosome, a complex macromolecular machinery composed of five small nuclear ribonucleoprotein particles (U1, U2, U4, U5, and U6 snRNP) and a large number of auxiliary proteins [3]. Alternatively, a small proportion of introns $(\approx 1 \%)$ are processed by the minor spliceosome comprising U11, U12, U4atac/U6atac, and U5 snRNPs [4]. The main spliceosome mediates the recognition of short consensus sequences defining the $5^{\prime}(\mathrm{GU})$ and $3^{\prime}$ (AG) splice sites (ss) and catalyses the two transesterification reactions necessary for the junction of exons and removal of introns [5]. Beside the conserved dinucleotide sequence that marks the ss, exon-intron junctions are not characterized by a stringent consensus and their short and degenerate nature is not sufficient to ensure perfect recognition by the spliceosome. Thus, additional factors are required to assist the spliceosome in its critical and essential function.

The activity of the spliceosome is regulated by both cisacting sequences on the pre-mRNA and transacting factors, which may enhance or inhibit both recognition of the ss and splicing catalysis [5]. The cis-acting regulatory elements are classified according to their location and activity into exonic and intronic splicing enhancers (ESEs and ISEs, resp.) or silencers (ESSs and ISSs, resp.) [6]. These sequence elements are recognized by transacting RNA binding proteins (RBPs), which in turn promote or inhibit spliceosome assembly and activity. Two main classes of RBPs that regulate splicing by binding to these cis-acting regulatory elements are the Ser/Arg rich (SR) proteins, which mainly exert a positive regulation on the spliceosome, and the heterogeneous nuclear ribonucleoproteins (hnRNPs), which often act antagonistically and inhibit splicing [7].

Chromatin signatures on the template DNA also participate in splicing regulation. Higher levels of nucleosome occupancy and specific histone modifications, such as trimethylation of H3K36, were found to be enriched in exons [8]. These observations suggest that epigenetic marks may facilitate exon recognition during splicing, perhaps by slowing down 
RNA polymerase II (RNAPII) in proximity of exons. Indeed, splicing largely occurs cotranscriptionally when the nascent pre-mRNA is still bound to the DNA template [9] and is likely affected by the elongation rate of the polymerase [10]. In addition, some splicing factors (SFs) interact with chromatinbinding proteins (i.e., MRG15, Gcn5, CHD1, and HP1 $\alpha$ ) and are recruited to histone marks enriched nearby exons, thereby modulating their selection $[10,11]$.

An additional layer of complexity to the splicing process is provided by the presence of exons characterized by even weaker elements defining exon-intron boundaries. Although this feature makes these exons weaker, it also represents a flexible resource for the gene as it allows their variable inclusion into mature transcripts through the alternative splicing (AS) process. Indeed, through differential assortment of weak or variable exons, a gene can yield multiple mRNA splice variants, potentially encoding proteins with different or even opposite function and/or displaying different patterns of spatial/temporal expression $[6,12]$.

The advent of high-throughput sequencing technologies has revealed the unexpected pervasive nature of AS. It is now clear that the vast majority of higher eukaryotic genes undergo AS $[13,14]$. In some cases, the combinatorial nature of AS allows a single gene to encode for up to thousands of mRNA variants. This extreme flexibility of the splicing process contributes to the great expansion of the coding potential and plasticity of the genome $[15,16]$. In support of this notion, eukaryotic cells have been documented to promptly modulate their splicing program in response to different intra- and extracellular cues [17], thus making AS a key tool to fine-tune gene expression. AS plays a pivotal role in controlling core cellular processes, such as proliferation, metabolism, and apoptosis, and fundamental physiological decisions, such as maintenance of pluripotent state or induction of a specific differentiation lineage [18]. Nevertheless, although AS represents a key tool to control gene expression in higher organisms, the extreme flexibility and multilayer nature of its regulation render it error prone and susceptible to alterations that threatens the maintenance of cellular homeostasis. As a proof of this concept, aberrant regulation of AS contributes to the onset or progression of several human diseases, including cancer $[19,20]$.

In the last decade, high-throughput analyses of transcriptomes have highlighted widespread alterations of AS patterns in human cancer $[21,22]$. When identified, the causes of these alterations were attributed to almost all the regulatory steps controlling AS [23-25]. Mutations in the cis-acting splicing regulatory elements, altered expression of SFs, and aberrant regulation of proteins and signalling pathway regulating their activity have all been documented in cancer cells and identified as factors promoting oncogenic splice variants and contributing to neoplastic transformation or later stages of carcinogenesis $[23,24]$. Thus, cancer cells can rapidly adapt to stimuli received from both extracellular and intracellular cues by finely regulating AS in order to shape gene expression. Herein, we will discuss examples of how AS contributes to the enhanced adaptation capability of cancer cells towards the adverse conditions occurring during the tumorigenic process or triggered by therapeutic intervention.

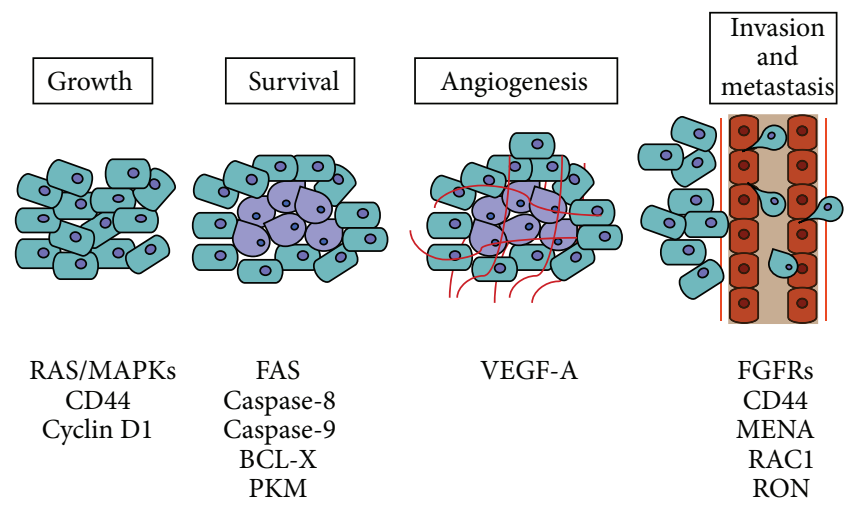

FIGURE 1: AS events that contribute to the adaptive response of cancer cells during tumorigenesis. Most relevant cancer-related genes undergoing AS misregulation are shown below the key events of tumoral transformation.

\section{Functional Impact of Splicing in Cancer Adaptation}

Cancer is a complex disease associated with a variety of genetic and epigenetic aberrations. As illustrated by Hanahan and Weinberg [26], during carcinogenesis cells acquire ten common traits: sustained proliferative signalling, resistance to death, evasion from growth suppressors, ability to invade normal tissues and metastasize, replicative immortality, induction of angiogenesis, genetic diversity generated by genome instability, inflammation, reprogramming of energy metabolism, and escape from immune destruction. A number of studies have now documented that aberrant regulation of AS in cancer cells contributes to many of these traits by allowing the production of oncogenic splice variants from multiple genes (Figure 1). Specific splice variant signatures are strongly associated with particular types of cancers, representing valuable diagnostic and prognostic markers $[27,28]$. Although their functional/mechanistic roles are still largely uncharacterised, these splice variants likely contribute to the acquisition of therapeutic resistance and to the increased adaptability of cancer cells to adverse environments. Herein, we will review some of the most important cancer-related AS events that play a functional role in the adaptation process set in motion by a tumour cell during both the early stages of development and progression of the pathology (Figure 2). Although cancer cells do not act "on purpose," we present a figurative writing style to stress the dynamic nature of cancer cell adaptation.

2.1. Sustained Proliferative Signalling. A critical feature of tumorigenesis is uncontrolled cell proliferation, including the ability to grow in the absence of external stimuli. This skill is acquired through a myriad of abnormal modifications of growth factor signalling cascades and expression of their messengers and effectors. It is therefore not surprising that the powerful combinatorial effect conferred by AS is hijacked by cancer cells to increase the expression of isoforms whose activity promotes and sustains cell proliferation. 


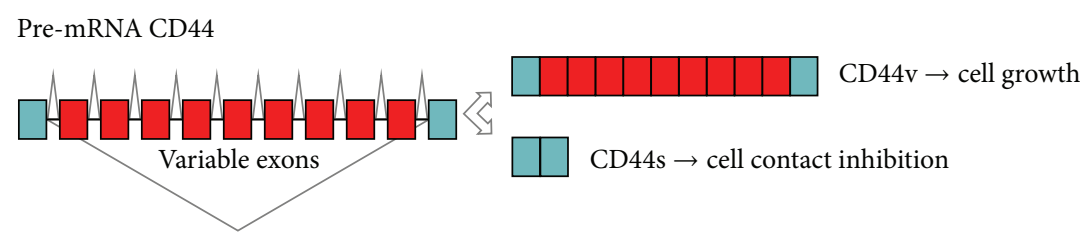

Pre-mRNA BCL-X

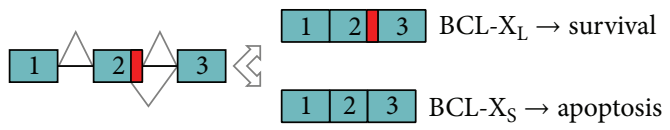

Pre-mRNA VEGF

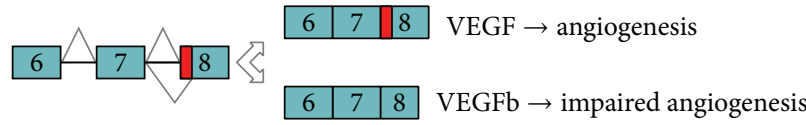

Pre-mRNA RON

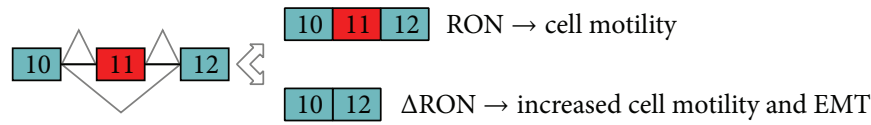

FIGURE 2: AS events that characterize specific phases of tumour occurrence and development. Green boxes and red boxes indicate constitutive and variable exons, respectively. Black lines indicate the intron sequences. The splice variants produced and their cellular functions are illustrated to the right of the gene schematic representation.

Homeostasis of growth control in cancer is often disrupted by constitutive activation of the RAS/MAPKs signalling pathway that plays a central role in cell proliferation, differentiation, and migration. Aberrant RAS activity may occur by several regulatory mechanisms that disrupt negative feedback loops or establish aberrant positive feedback loops in the pathway. The misregulation of AS of genes involved in the RAS pathway contributes to its activation in cancer, thus enhancing cell proliferation. An example is represented by the RAS-activated A-RAF kinase. AS of $A-R A F$ is modulated by the splicing factor (SF) hnRNP A2, which represses the production of a short dominant-negative isoform in favour of the full-length transcript. Aberrant regulation of this splicing event leads to constitutive activation of the RAS/MAPKs pathway, cellular transformation, and increased proliferation [29]. Similarly, AS of $B-R A F$ (V600E), a mutation present in $50 \%$ of metastatic melanomas, might result in being critical for this cancer and its treatment. A recent study involving experimental cell culture models and patient samples showed the existence of B-RAF (V600E) splice isoforms that lack the RAS binding domain and promote resistance to chemotherapy [30].

Hyperactivation of transmembrane receptors upstream of RAS can also contribute to favouring cancer-related AS events through positive feedback loops that modulate the activity of specific SFs. A typical example is represented by the epidermal growth factor receptor (EGFR), a tyrosine kinase receptor that plays a central role in cell proliferation and motility. EGFR pre-mRNA is alternatively spliced to generate a variant lacking exon $4(\mathrm{EGFR} \Delta 4)$. Skipping of this exon yields a receptor that is constitutively active and promotes proliferation. Notably, the EGFR $\Delta 4$ isoform is abundantly expressed in several cancers, such as glioma, prostate, and ovarian cancer [31]. Furthermore, a recent work documented that an active EGF signalling per se induces a massive reprogramming of AS. This effect was attributed to AKT-induced nuclear translocation of the SR protein kinase 1 (SRPK1). AKT binding to SRPK1 induces its autophosphorylation and dissociation from the HSP70 chaperone, which normally holds SRPK1 into the cytoplasm, thus favouring its nuclear translocation [32]. Once in the nucleus, SRPK1 can phosphorylate SR proteins and modulate the splicing pattern of several genes [32]. Since SRPK1 is usually localized in the cytoplasm in the absence of an extracellular signal and phosphorylates shuttling SR proteins in this cellular compartment $[33,34]$, stress signals might expand the effect of SRPK1 activation to the nucleus and influence also SR proteins that mostly reside in this compartment.

AS of the CD44 gene also serves as a critical mechanism for a feed-forward loop that sustains activation of RAS/ MAPK signalling [35]. CD44 is a transmembrane glycoprotein mediating the response of cells to their cellular microenvironment. CD44 is expressed in most tissues, where it functions in lymphocyte homing, adhesion, migration, and regulation of cell growth [36]. This variety of roles is favoured by the existence of multiple CD44 splice variants. The CD44 gene is composed of 10 constitutively spliced exons and 10 variable exons, residing between constitutive exons 5 and 6 . Upon mitogenic activation, the RAS/MAPK pathway positively regulates the activity of SAM68 and SRm160, two SFs that promote inclusion of variable exons in CD44 [37, 38]. The newly synthesized CD44v6 isoform, containing variable 
exon 6, forms complexes with receptor tyrosine kinases (RTKs) that promote RAS/MAPK activation and cell cycle progression [35].

Another oncogenic AS event that sustains uncontrolled proliferation of cancer cells affects the cyclin D1 (CCND1) protooncogene. Cyclin D1 associates with the cyclin-dependent kinase 4 (CDK4) to drive progression through the G1 phase of the cell cycle. Importantly, cyclin D1 expression is often deregulated in cancer cells $[39,40]$. This gene encodes for two alternative transcripts: the common cyclin Dla isoform and the prooncogenic cyclin D1b isoform. In prostate epithelial cells, the canonical cyclin Dla isoform is involved in a negative feedback loop that controls proliferation. Cyclin Dla interacts with and represses the transcriptional activity of the androgen receptor (AR), which orchestrates the proliferation and activity of prostate cells [41]. By contrast, although the cyclin D1b isoform is capable of driving the G1/S transition of the cell cycle and to interact with AR, it does not repress its transcriptional activity, thereby interrupting this negative feedback [41]. Notably, two SFs that are often upregulated in cancer cells $[42,43]$, SRSF1 and SAM68, promote cyclin D1b splicing in prostate cancer cells [44, 45]. SAM68dependent regulation of cyclin Dlb splicing represents another clear example of how activated signalling pathways modulate cancer-related AS events by influencing the activity of specific SFs. Indeed, activation of the RAS/MAPKs pathway enhanced SAM68 binding affinity for cyclin D1 RNA and SAM68-dependent cyclin D1b splicing, whereas SAM68 phosphorylation by SRC-family kinases (SFKs) counteracted these activities [45]. Thus, these studies indicate how upregulation of two oncogenic SFs can unleash prostate cancer cells from the cyclin Dla/AR negative feedback that limits excessive proliferation of the epithelial cells in the normal organ.

2.2. Induction of Angiogenesis. Angiogenesis is the physiological process yielding new blood vessels. Neoangiogenesis normally occurs during embryogenesis and fetal development in response to the need for oxygen and nutrients of the growing mass of cells forming new tissues and organs. A similar situation occurs during tumorigenesis, when cancer cells begin to proliferate within a steady-state adult tissue. Growth of the tumour mass depletes the host tissue of nutrients and oxygen, causing starvation and promoting the formation of new vessels as an adaptive response. Tumour-associated neoangiogenesis provides cancers cells with access to blood circulation, thus facilitating tumour growth.

The main growth factors promoting angiogenesis are the vascular endothelial growth factors (VEGF) [46]. In humans, the VEGF family consists of five ligands and three signalling receptors. The ligands, VEGF-A-D and placental growth factor, are all alternatively spliced to yield isoforms with opposite or, in some cases, unknown function. Since VEGF-A AS is altered in a number of cancers, such as metastatic melanoma, neuroblastoma, and renal, prostate, colorectal, and bladder cancers [47], this gene is also the most studied of the family.

The alternative splice variants of VEGF-A exert different effects on tissue and tumour growth due to their opposing effects on angiogenesis. Several regulatory mechanisms that are critical for the splicing of this gene have now been identified. The best-known one consists in alternative usage of two $3^{\prime}$ ss in VEGF-A exon 8 [48]. SRSF1 and SRSF5 (SRp40) promote usage of the proximal $3^{\prime}$ ss, thus favouring the production of mRNAs encoding proangiogenic proteins [49]. By contrast, SRSF6 (SRp55) and SRSF2 (SC35) facilitate the selection of the distal $3^{\prime}$ ss, resulting in production of the antiangiogenic VEGFb isoform [49].

Signalling pathways evoked in cancer cells by the surrounding environment can mediate the balance between antiangiogenic and proangiogenic VEGF isoforms [50, 51]. Such regulation occurs either by direct control of their phosphorylation status by signalling kinases or by indirectly regulating splicing factor kinases involved in their posttranslational modifications. An example of indirect regulation is illustrated by insulin-like growth factor-1- (IGF-1-) mediated activation of protein kinase $\mathrm{C}$ (PKC) signalling, which in turn positively regulates SRPK1-dependent phosphorylation of SRSF1 and SRSF1-dependent VEGF-A AS [51]. A similar regulatory mechanism is also observed in prostate cancer, where selective upregulation of proangiogenic VEGF is under the direct control of SRPK1-regulated SRSF1 activity [50]. Importantly, genetic or pharmacological interference with SRPK1 activity caused a switch in the expression of proangiogenic towards antiangiogenic VEGF splice isoform, resulting in decreased microvessel density and reduced tumour growth [50]. Thus, the upregulation of SRPK1 and SRSF1 activity frequently observed in human cancers might contribute to the ability of the tumour mass to promote neoangiogenesis and redirect the blood stream towards itself. Alternatively, SRSF1-dependent VEGF-A AS may be indirectly regulated by the transcription factor WT1, encoded by the Wilms' tumour gene (WT1) [52]. It was shown that WT1 represses the transcription of SRPK1 by directly binding to its promoter. This effect results in reduced SRPK1-dependent SRSF1 phosphorylation and inhibition of the production of prooncogenic VEGF isoform. Importantly, the authors demonstrated that in WT1 mutant cells SRPK1 is highly expressed, SRSF1 is hyperphosphorylated, and VEGF prooncogenic isoforms are abundant, causing abnormal angiogenesis [52].

Recent evidence describes other novel regulatory circuits underlying the $V E G F-A$ gene regulation that do not depend on the activity of SR proteins and/or on different usage of the $3^{\prime}$ ss in exon 8. For instance, VEGF-A AS is modulated by the alternatively spliced isoforms of the splicing factor $\mathrm{T}$ cell intracellular antigen (TIA-1). AS of TIA-1 leads to expression of a truncated protein, called short TIA-1 (sTIA-1) in some cancer cells [53]. sTIA-1 competes with the binding of fulllength TIA-1 to VEGF-A mRNA, thus favouring the production of the prooncogenic VEGF-A isoform, angiogenesis, and tumour growth in animal models. Notably, sTIA-1 expression positively correlates with advanced tumour stage in colorectal carcinoma (CRC) patients, supporting its prooncogenic function [53].

In addition to the well-studied antiangiogenic (VEGFb) and proangiogenic (VEGF) $V E G F-A$ isoforms, a novel isoform named VEGF-A “extended” (VEGFAx), which displays antiangiogenic activity, was recently described [54]. In line 
with its inhibitory function on angiogenesis, VEGFAx expression levels are reduced in high-grade CRC tumours with respect to normal human colon mucosa. This isoform is produced by an uncommon regulatory mechanism, called programmed translational readthrough (PTR). This process is due to the presence of a cis-acting element that directs protein translation to continue beyond the canonical stop codon, with translation stopping at an alternative downstream stop codon. A recognition element for hnRNP A2/B1 was identified in the Ax region and loss of this recognition site, by either mutation of the sequence or knockdown of hnRNP A2/B1, reduced expression of VEGFAx [54].

AS of the VEGF-A gene may also be affected by epigenetic mechanisms. Chromatin features can directly affect splicing outcome by physically coupling the transcription machinery with the splicing apparatus via chromatin-binding adaptor proteins. The latter recognize exons or introns enriched in particular histone modifications and, in turn, recruit splicing regulators to nascent pre-mRNAs [10]. Using a high-throughput screen, VEGF- $A$ was identified as a main target for chromatin-mediated AS regulation [55]. The authors showed that $\mathrm{H} 3 \mathrm{~K} 9$ methylation operated by the methyltransferase EHMT2 favours recruitment of the chromatin-binding protein HP1 $\gamma$ and its associated partner SRSF1 with the VEGF pre-mRNA, thus modulating its AS.

These examples illustrate the complexity of the regulation underlying VEGF-A pre-mRNA processing and translation and highlights how this process amplifies the escape routes available for cancer cells to adapt to an adverse environment.

2.3. Invasion and Metastasis. More than $90 \%$ of cancerrelated deaths are due to metastasis and spread of cancer cells to multiple tissues and organs. The ability to form metastasis is probably the most complex task for cancer cells, which need to migrate from the primary tumour, intravasate, survive in blood, extravasate, and colonise different new environments. This implies an incredible phenotypic plasticity, which is largely due to a process called epithelial to mesenchymal transition (EMT) and the reverse, mesenchymal to epithelial transitions (MET) [56]. Through EMT, epithelial cells undergo an extensive reorganization of cytoskeletal architecture, with loss of intercellular junctions and cell polarity and acquisition of an elongated, fibroblast-like shape, thus acquiring invasive capabilities. EMT physiologically pertains to embryogenesis, when cells migrate to shape new organs, but it is adopted by cancer cells to generate metastases.

The ability of cancer cells to undergo EMT relies on the activation of a specific gene expression program in response to extracellular cues. Several interconnected regulatory networks drive EMT and modulation of any of them elicits profound effects on the others. The most extensively studied network is built around the transcription factors SNAIL, SLUG, ZEB1/2, and TWIST. Cues from the tumour microenvironment favour the expression of these factors and trigger a global change in gene expression that underlies EMT [57]. Nevertheless, other regulatory layers, including co- and posttranscriptional control by AS and small noncoding RNAs, interconnect with the transcriptional program and in some case can substitute or activate it, setting in motion critical aspects of EMT-associated phenotypic changes [57].

Many EMT-related genes generate AS variants encoding for proteins with essential functions in EMT and this topic has been recently reviewed elsewhere [57-59]. Herein, we wish to highlight few of the most relevant and well-described events. During EMT, several adhesion molecules specific of epithelial or mesenchymal cells are regulated through AS, such as CD44, p120-catenin (CTNND1), and MENA (ENAH) proteins [57]. For instance, AS of the CD44 gene is tightly regulated during EMT in breast cancer cells. CD44 AS is governed by the epithelial splicing regulatory protein 1 (ESRP1), a SF that stimulates inclusion of variable exons $(\mathrm{CD} 44 \mathrm{v}$ isoforms). During EMT, ESRP1 levels drastically decrease, leading to the upregulation of the standard isoform (CD44s), which contributes to the formation of EMT-associated recurrent breast cancer in mice [60]. ESPR1 also positively regulates the production of the epithelial isoform MENA1la, which inhibits the migratory ability of breast cancer cells and is able to counteract the invasive activity of the mesenchymal MENA $\Delta \mathrm{v} 6$ isoform of the same gene [61].

The ESRP family members (ESRP1 and ESRP2) are so far the only known SFs exhibiting epithelial cell-type-specific expression and that undergo pronounced changes in expression during EMT $[62,63]$. High-throughput experimental approaches revealed a high-affinity ESRP-binding motif (with UGG as a core motif) and a predictive "RNA map" that governs ESRP1/2 activity [64]. Importantly, downregulation of ESRP proteins during EMT affects splicing of a large number of these target genes, indicating that ESRPs are key players in this cancer-related cellular transition.

Other tissue-specific and more ubiquitously expressed SFs, such as the RBP FOX1 homologue (RBFOX), CUGBP Elav-like family (CELF), muscleblind-like protein (MBNL), SR proteins, and hnRNPs, also play a role in EMT [58]. For instance, hnRNP A1 has been recently implicated in the induction of the RAClb isoform of the GTPase RAC1 [65], which is known to induce EMT through generation of reactive oxygen species (ROS) and induction of SNAIL expression [66]. hnRNP A1 negatively regulates RAClb splicing by binding to RAC1 alternative exon $3 \mathrm{~b}$ and inhibiting its inclusion; treatment with matrix metalloproteinase-3 (MMP-3) inhibits hnRNP Al binding to exon $3 \mathrm{~b}$, thus relieving its repressive activity and favouring RAClb splicing in mammary epithelial cells [65]. Conversely, in colorectal cells RAC1b splicing is positively regulated by the SR protein SRSF1 in a SRPK1regulated manner [67]. Recent evidence shows indeed that the knockdown of SRPK1 or inhibition of its catalytic activity reduced phosphorylation and subsequent translocation of SRSF1 to the nucleus, limiting its availability to promote the inclusion of alternative exon $3 \mathrm{~b}$ into the RAC1b pre-mRNA [67]. Thus, although a direct competition between SRSF1 and hnRNP Al in RAClb splicing regulation has not been demonstrated, it is tempting to speculate that epithelial or mesenchymal phenotype of a cancer cell could be modulated by the balance in the activity of these SFs and the consequent effect on RAClb splicing.

SRSF1 also regulates the splicing of the tyrosine kinase receptor RON by inhibiting inclusion of exon 11 [68]. 
The resulting $\triangle \mathrm{RON}$ isoform is unable to undergo proteolytic cleavage, rendering the protein constitutively active and conferring increased motility to cancer cells $[68,69]$. Importantly, cancer cells modulate the expression levels of SRSF1, splicing of the $\triangle \mathrm{RON}$ isoform, and induction of EMT in response to external cues from the surrounding environment. This process is orchestrated by a splicing cascade relying on phosphorylation/activation of the SAM68 by the extracellular regulated protein kinases (ERK1/2). Once activated, SAM68 promotes inclusion of a cryptic intron in the $3^{\prime}$ untranslated region of SRSF1 mRNA, thus inhibiting its degradation by nonsense mediated decay (NMD) [70].

Altogether, these observations indicate that AS plays a major role in EMT by establishing a specific program of splice variants of genes important for epithelial and mesenchymal cell morphology and motility. These observations raise the intriguing possibility that abnormal changes in splicing can steer cancer cells towards malignant progression through a partial EMT, without the need for canonical transcriptional reprogramming.

2.4. Resisting Cell Death. Apoptosis (also called programmed cell death) is a death process characterized by shrinkage of the cell and its nucleus. The apoptotic machinery is composed of both upstream regulators and downstream effector components. These players receive and integrate extracellular or intracellular cell death-inducing signals, giving rise to extrinsic and intrinsic apoptotic programs [26]. Both pathways culminate in a proteolytic cascade exerted by caspases. Once an insult hits a cell, it is the balance between pro- and antiapoptotic factors that determines cell fate.

Although apoptosis serves as a natural barrier to eliminate cells that develop aberrant features, transformed cells have developed a variety of strategies to limit or circumvent it. One of such strategies consists in modulation of AS to shift expression from pro- to antiapoptotic isoforms of several genes. Below, we will summarize some examples of apoptosisrelated AS events that occur in the tumour microenvironment. As can be inferred from the list, each AS event is finely regulated by many SFs exhibiting synergistic or opposing functions. The cancer cell exploits the cooperation or competition between them to establish regulatory mechanisms that favour the production of the splicing isoform suitable for survival.

The death receptor FAS (an upstream regulator that receives extracellular death signals induced by the FAS ligand) and CASP9 and CASP8 (initial executioners of apoptosis) genes are regulated by AS, giving rise to splice isoforms with pro- or antiapoptotic roles. For instance, inclusion or skipping of FAS exon 6, respectively, generates two functionally distinct receptors, a membrane-bound protein with proapoptotic function and a soluble form with antiapoptotic function [71, 72]. TIA-1, TIAR-1 (TIA-1 related) [73], and EWS (Ewing sarcoma protein) [74] positively regulate FAS splicing by favouring the assembly of the spliceosome on the $5^{\prime}$ and $3^{\prime}$ ss of exon 6 , resulting in the generation of the proapoptotic isoform. By contrast, PTB/hnRNP I [73], RBM5 [75], HuR [76], and hnRNP C1/C2 [77] negatively regulate exon 6 splicing in favour of the antiapoptotic FAS isoform.
Caspase- 9 is the most studied family member in terms of AS. The CASP9 gene generates two splice variants, the proapoptotic caspase-9a and the antiapoptotic caspase-9b, which differ for the inclusion or exclusion of a four-exon cassette (exons 3, 4, 5, and 6), respectively [78, 79]. SRSF1 promotes the inclusion of the exon cassette contributing to the generation of caspase-9a proapoptotic isoform in non-small cell lung cancer (NSCLC) cells [80]. However, constitutive activation of the PI3K/AKT pathway in these cells repressed this activity [80]. On the other hand, hnRNP L promotes skipping of the exon cassette to generate the antiapoptotic caspase-9b protein [81]. Interestingly, the expression level and the phosphorylation status of hnRNP L strongly influence the outcome of this AS event. Overexpression of hnRNP L in NSCLC cells, but not in nontransformed cells, lowers the caspase-9a/9b ratio, favouring the oncogenic isoform. The physiological relevance of this mechanism was confirmed by the complete loss of tumorigenic capacity in a mouse xenograft model of NSCLC cells depleted of hnRNP L [81].

The cancer-restricted role of hnRNP L in caspase-9 AS is apparently due to NSCLC-specific phosphorylation of hnRNP L on Ser52, suggesting that cancer cell developed a device to switch an ubiquitous RBP into a prooncogenic protein through a specific posttranslational modification.

Many other apoptosis-related genes are also subjected to AS regulation. The $B C L-X$ (BCL2L1) gene contains 3 exons and encodes two splice variants [82]. Two alternative $5^{\prime}$ ss are present in exon 2: selection of the canonical one at the end of the exon yields the long, antiapoptotic variant BCL- $\mathrm{X}_{\mathrm{L}}$, whereas selection of the distal one located upstream in the exon produces the short, proapoptotic variant BCL- $\mathrm{X}_{\mathrm{S}}$ [82]. Several SFs have been shown to modulate $B C L-X$ splicing. HnRNP H, F, and I (PTB) [83, 84], SAM68 [85], the RBPs RBM25 [86], and RBM11 [87] were all shown to promote splicing of the proapoptotic BCL- $\mathrm{X}_{\mathrm{S}}$ variant. By contrast, the SFs SAP155 [88], SRSF9 [89], hnRNP K [90], and SRSF1 $[85,91]$ enhance splicing of the antiapoptotic BCL- $\mathrm{X}_{\mathrm{L}}$. The balance of BCL-X isoforms is affected in a large number of cancer cell lines and human cancer samples, and fine-tuned regulation of this AS event can determine the cell fate in response to various stresses $[85,92,93]$.

These examples highlight how different families of SFs are employed by cancer cells to coordinate splicing regulation and to promote cell survival in response to the hazards imposed by the variable environmental conditions, gaining an advantage with respect to nontransformed cells.

2.5. Deregulating Cellular Energies. The uncontrolled cell proliferation that characterizes cancer cells involves adjustments of energy metabolism in order to favour a rapid growth and division of tumour cells even in adverse microenvironments. Under aerobic conditions, cells produce energy via glycolysis in the cytosol (this reaction allows the conversion of glucose to pyruvate) and then via oxidative phosphorylation in the mitochondria (this reaction allows the conversion of pyruvate to carbon dioxide). Under anaerobic conditions, glycolysis is favoured compared to oxygen-consuming mitochondrial oxidative phosphorylation. Cancer cells, however, primarily use glycolysis, by reprogramming their glucose 
metabolism and energy production regardless of oxygen supply. This cancer-related process, called "aerobic glycolysis," was already discovered in 1930 by Warburg $[94,95]$. The efficiency of ATP production insured by glycolysis is lower than that provided by mitochondrial oxidative phosphorylation. However, an increased glycolysis provides advantages to cancer cells by allowing a more efficient utilization of glycolytic intermediates in other biosynthetic pathways that favour proliferation also in presence of limited amounts of nutrients [96]. This reliance on glycolysis can be further accentuated under the hypoxic conditions occurring within the growing tumour mass.

AS of key metabolic enzymes partially governs the metabolic switch that characterizes cancer cell metabolism. A wellstudied example is that of pyruvate kinase (PKM), an enzyme that catalyses the conversion of phosphoenolpyruvate (PEP) to pyruvate [97]. The PKM gene encodes two alternative splice variants through usage of mutually exclusive exons [97]. The PKM1 isoform, produced when exon 9 is included in the mature transcript, is normally expressed in adult life and stimulates mitochondrial oxidative phosphorylation. PKM2, generated by inclusion of exon 10, is exclusively expressed during embryonic development and promotes aerobic glycolysis. However, PKM2 is typically reexpressed in cancer cells where it confers oncogenic features [97-99]. Indeed, replacement of PKM2 with PKM1 in lung tumour cells correlated with impaired tumour occurrence in mouse xenografts [97]. PKM splicing in cancer cells is modulated by hnRNP A1, hnRNP A2, and hnRNP I/PTB, which cooperate to promote splicing of PKM2 by binding to sequences flanking exon 9 and repressing its inclusion $[100,101]$. Notably, all three hnRNPs are overexpressed in several cancers $[23,24]$ and their expression can be coordinated by the oncogenic transcription factor MYC [101]. Thus, during neoplastic transformation upregulation of MYC activity and of these SFs might predispose the cell to alter its energy metabolism through modulation of PKM AS. This transition would render the cancer cell less susceptible to starvation and/or other unfavourable metabolic conditions occurring in the tumour microenvironment.

2.6. Chemotherapy Resistance. Surgery, radiation, and chemotherapeutic drugs are the standard approaches for cancer treatment. Radiation and chemotherapy mainly act by inducing cancer cell death. Although most tumours respond to chemotherapy at first, some cancer cells often survive treatments, expand, and acquire chemoresistance causing disease relapse. The mechanisms by which cancer cells adapt or are selected for their resistance to treatments vary with cancer type and from patient to patient. Most of these mechanisms causing chemotherapy resistance have been elegantly described elsewhere and mainly involve mutations and/or altered expression of genes and proteins [102]. Among these, AS participates in the process of acquired chemoresistance by controlling the expression of cancer-related splice variants that contribute to cancerous phenotype (Figure 3). Herein, we illustrate some examples of how AS allows cancer cells to adapt to tumour microenvironment, under conditions where normal cells would undergo cell death, and to overcome the chemotherapy-mediated selective pressures.

An interesting example of AS adaptive response driven by chemotherapy is provided by the switch from cyclin Dla to cyclin Dlb in breast cancer cells. Upon treatment of MCF7 cells with cisplatin and the estrogen receptor antagonists 4-hydroxy tamoxifen and ICI 182780, endogenous protein cyclin Dla expression is strongly reduced, whereas oncogenic cyclin D1b splice variant is maintained and confers chemoresistance [103].

The HER2-targeted therapy using trastuzumab is widely used for the treatment of patients with metastatic breast tumours overexpressing HER2, a member of EGFR family of receptor tyrosine kinases. Although the search for a somatic HER2 oncogenic mutation in HER2-amplified breast tumours has failed to identify a promising activating genetic lesion [104-106], the existence of HER2 isoforms that may influence trastuzumab response in breast tumours evidenced the key role of AS in chemoresistance [107-109]. A new HER2 splice variant (HER2 $\triangle 16)$ with potent transforming activity was detected in several HER2-overexpressing breast cancer cell lines $[108,109]$ and primary tumours $[107,109]$. Furthermore, the expression of HER2 $\Delta 16$ is a tumour-specific molecular event and the vast majority of women with expression of HER2 $\Delta 16$ develop locally disseminated node-positive breast cancer. Furthermore, tumour cell lines expressing HER2 $\Delta 16$ are resistant to the HER2-targeted therapy trastuzumab [110]. The critical effector of HER $2 \Delta 16$ tumorigenic properties is represented by SRC kinase. In fact, SRC kinase appears to function as a "master regulator" stabilizing HER $2 \Delta 16$ protein expression and coupling HER2 $\Delta 16$ to multiple mitogenic and cell motility pathways [110]. Cotargeting of HER2 $\Delta 16$ and SRC kinase with the single agent tyrosine kinase inhibitor dasatinib resulted in SRC inactivation, destabilization of HER2 $\Delta 16$, and suppressed tumorigenicity [110]. An important issue will be to characterize the cancer-specific splicing event leading to HER $2 \Delta 16$ expression in breast cells. Understanding these mechanisms might indeed offer therapeutic perspective to counteract the activity of this oncogenic splice variant in breast cancers with poor prognosis.

Another SF involved in drug resistance is SPF45, a $45 \mathrm{kDa}$ nuclear protein $[111,112]$. SPF 45 is highly expressed in numerous carcinomas including bladder, breast, colon, lung, ovarian, pancreatic, and prostate. Forced overexpression of SPF45 in HeLa cells demonstrated a 4-7-fold increase in resistance to doxorubicin. Ectopic SPF45 expression in the A2780 ovarian cancer cells induced a multidrug resistant phenotype, inducing 3-21-fold resistance to a variety of chemotherapeutics with differing mechanisms of action, including carboplatin, vinorelbine, doxorubicin, etoposide, mitoxantrone, and vincristine [111]. The mechanism underlying the multidrug resistant phenotype acquired upon SPF45 overexpression is still unknown but probably relies on misregulation of AS of its targets [113, 114]. Few splicing targets of SPF45 are currently known. SPF45 promotes the proapoptotic transmembrane receptor FAS pre-mRNA [114], but this activity is repressed by both mitogenic (ERK1/2) and stress-response (p38 and JUN N-terminal kinases) MAPKdependent phosphorylation in cancer cells [113]. SPF45 is 


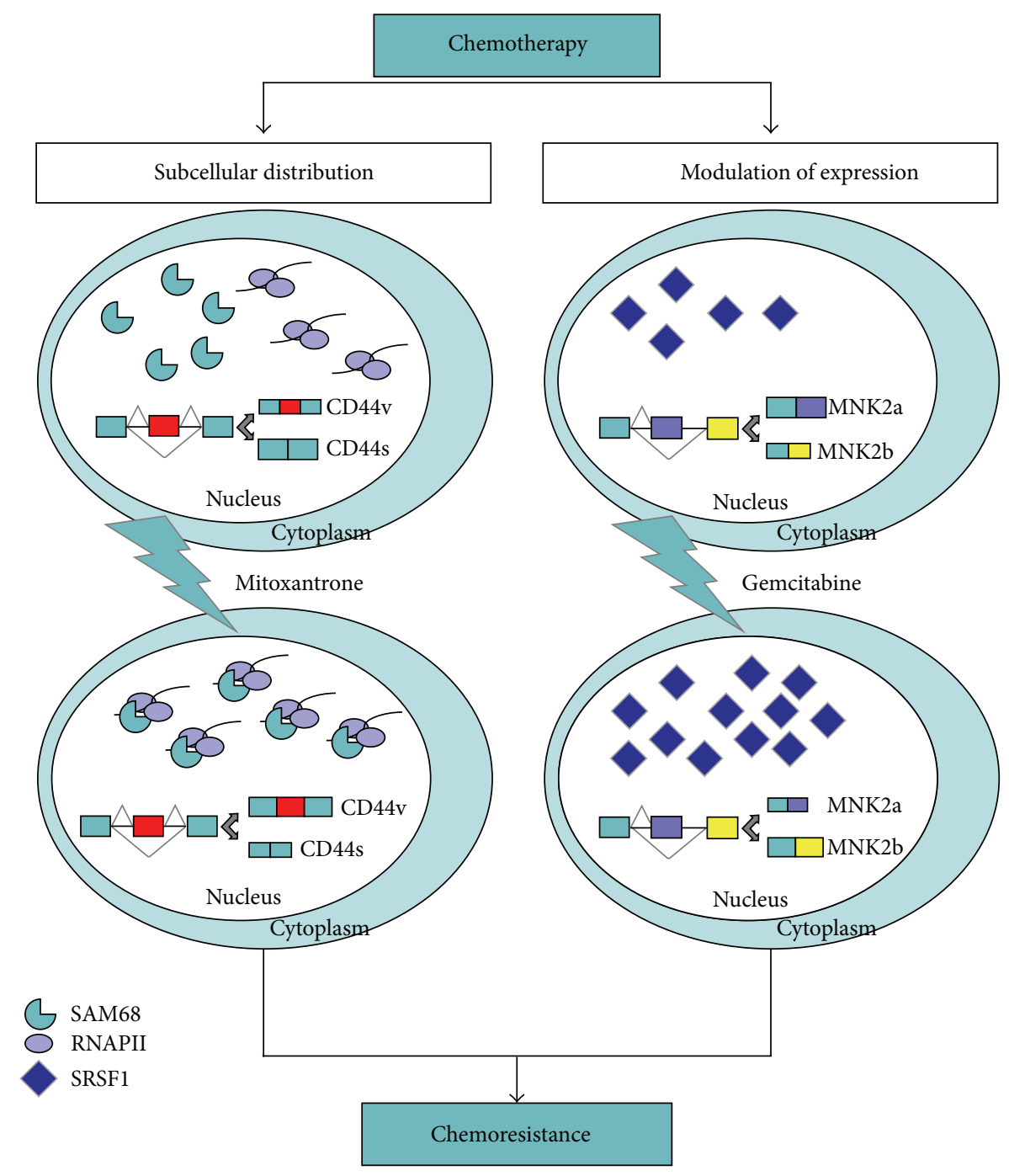

FIGURE 3: Regulation of chemoresistance via alternative messenger RNA splicing. Chemotherapy can affect the subnuclear distribution of SAM68 (left panel) and the expression of SRSF1 (right panel), thus modulating AS of cancer-related variants (CD44v and MNK2b, resp.) and contributing to chemoresistance. Green boxes indicate constitutive exons; red, yellow, and violet boxes indicate variable exons.

also phosphorylated by CLK1 on multiple serine residues and this posttranslational modification regulates alternative ss utilization by SPF45 and its intranuclear localization [115]. Furthermore, stable SPF45 overexpression in SKOV-3 cells induces enhancement of fibronectin 1 expression and regulates fibronectin 1 AS by enhancing inclusion of the EDA region into fibronectin transcripts [113]. Since inclusion of EDA region in fibronectin enhances the migratory capacity of embryonic cells and tissue, SPF45 overexpression might contribute to promote metastasis in vivo by modulating this AS event. Thus, full elucidation of the spectrum of AS events regulated by this $\mathrm{SF}$ in cancer cells might reveal pathways involved not only in acquisition of chemoresistance but also in other key oncogenic features.

Although a large spectrum of AS events associated with chemoresistance has been described [116-118], much less is known about the mechanisms activated during chemotherapy that result in the observed splicing changes. In this regard, genotoxic stress may cause a subcellular redistribution of many RBPs and/or modify their activity through posttranslational modifications as an attempt of the cancer cell to adapt to the hostile environment [119-121]. The subcellular localization of SAM68 and other RBPs was affected by treatment of prostate cancer cells with mitoxantrone (MTX), a topoisomerase II inhibitor used in chemotherapy, partially altering the cellular AS pattern [119]. Another regulatory mechanism through which tumour cells acquire resistance involves the modulation of expression of specific SFs or of cancer-related splicing variants and/or of their counterparts. Treatment of pancreatic ductal adenocarcinoma (PDAC) cells with gemcitabine induced the upregulation of SRSF1 that, in turn, regulates AS of mitogen activated protein kinase (MAPK) interacting kinase 2 (MNK2) in favour of MNK2b isoform [122]. SRSF1-dependent AS of MNK2b following gemcitabine treatment conferred increased resistance of PDAC cells to chemotherapeutic drug, identifying a novel chemotherapymediated adaptation response through AS in PDAC cells [122]. Notably, a recent report showed that MNK2a behaves as 
tumour suppressor in breast cancer, whereas the alternative MNK2b splice variant was prooncogenic [123]. Thus, it appears that upregulation of SRSF1 in response to genotoxic stress confers resistance to treatments by switching this splicing event in favour of the prooncogenic MNK2b variant.

These observations suggest that AS changes induced by chemotherapeutic treatment represent an important sideeffect, which may contribute to therapy resistance. These aspects need to be taken into account for the development of new therapeutic protocols that could exploit the combined usage of canonical chemotherapy with novel pharmaceutical tools targeting the adaptive splicing response associated with treatments.

\section{Concluding Remarks}

As shown by the several examples illustrated in this review, AS plays a key role in the rearrangement of gene expression, thus enabling cancer cells to adapt to the adverse conditions encountered during the transformation process and to evade different therapeutic approaches. At the same time, these observations suggest that the splice variants aberrantly expressed by cancer cells might represent suitable targets for the development of new antitumor therapies, in particular those whose prognostic or diagnostic values have already been demonstrated [124]. Redirecting aberrant splicing events or inhibiting the activity of oncogenic splice variants can represent a valuable approach to increase cancer cells sensitivity to canonical chemotherapies, which could be exploited in new combined therapies. As an example, susceptibility of NSCLC cells to different chemotherapeutic drugs can be enhanced by RNA interference of the expression of the antiapoptotic splice variant caspase-9b of the CASP9 gene [125]. Notably, one of the advantages of therapeutically targeting alternative splice variants is the possibility to act on two different fronts: on one hand, therapies targeting the specific activity of the oncogenic splice variant could be developed; on the other hand, the mechanisms driving the aberrant splicing event could also be targeted. In light of this, great interest has arisen for studies exploiting antisense oligonucleotide (ASO) to redirect splicing of tumoral variants towards a nontumoral isoform. This approach has been recently shown to be possible for the PKM2/PKM1 [126] and the BCL-X $\mathrm{L}_{\mathrm{S}}$ [93] splicing switch. The high therapeutic value of the ASO approach is strongly supported by recent studies demonstrating the good bioavailability and efficacy of an ASO redirecting SMN2 splicing for the treatment of SMA animal models [127].

Thus, it is certainly possible to envision the development in the near future of new personal anticancer therapies targeting the specific splicing-alterations of each patient, whose identification will be ensured by the novel and rapidly evolving high-throughput sequencing techniques that allow genome-wide profiling of cellular transcriptomes, even at a single cell-resolution [21].

\section{Conflict of Interests}

The authors declare that there is no conflict of interests regarding the publication of this paper.

\section{Acknowledgments}

This work was supported by the Association for International Cancer Research (AICR) (Grant no. 12-0150); Associazione Italiana Ricerca sul Cancro (AIRC) (Grant no. 14581); and Fondazione Santa Lucia Ricerca Corrente.

\section{References}

[1] I. B. Rogozin, L. Carmel, M. Csuros, and E. V. Koonin, "Origin and evolution of spliceosomal introns," Biology Direct, vol. 7, article 11, 2012.

[2] M. J. Moore and N. J. Proudfoot, "Pre-mRNA processing reaches back to transcription and ahead to translation," Cell, vol. 136, no. 4, pp. 688-700, 2009.

[3] C. L. Will and R. Lührmann, "Spliceosome structure and function," Cold Spring Harbor Perspectives in Biology, vol. 3, no. 7, pp. 1-2, 2011.

[4] A. A. Patel and J. A. Steitz, "Splicing double: insights from the second spliceosome," Nature Reviews Molecular Cell Biology, vol. 4, no. 12, pp. 960-970, 2003.

[5] A. G. Matera and Z. Wang, "A day in the life of the spliceosome," Nature Reviews Molecular Cell Biology, vol. 15, no. 2, pp. 108-121, 2014.

[6] D. L. Black, "Mechanisms of alternative pre-messenger RNA splicing," Annual Review of Biochemistry, vol. 72, pp. 291-336, 2003.

[7] A. Busch and K. J. Hertel, "Evolution of SR protein and hnRNP splicing regulatory factors," Wiley Interdisciplinary Reviews: RNA, vol. 3, no. 1, pp. 1-12, 2012.

[8] S. Schwartz and G. Ast, "Chromatin density and splicing destiny: on the cross-talk between chromatin structure and splicing," EMBO Journal, vol. 29, no. 10, pp. 1629-1636, 2010.

[9] A. Pandya-Jones and D. L. Black, "Co-transcriptional splicing of constitutive and alternative exons," $R N A$, vol. 15, no. 10, pp. 1896-1908, 2009.

[10] R. F. Luco, M. Allo, I. E. Schor, A. R. Kornblihtt, and T. Misteli, "Epigenetics in alternative pre-mRNA splicing," Cell, vol. 144, no. 1, pp. 16-26, 2011.

[11] S. F. de Almeida and M. Carmo-Fonseca, "Reciprocal regulatory links between cotranscriptional splicing and chromatin," Seminars in Cell \& Developmental Biology, vol. 32, pp. 2-10, 2014.

[12] M. Chen and J. L. Manley, "Mechanisms of alternative splicing regulation: insights from molecular and genomics approaches," Nature Reviews Molecular Cell Biology, vol. 10, no. 11, pp. 741754, 2009.

[13] Q. Pan, O. Shai, L. J. Lee, B. J. Frey, and B. J. Blencowe, "Deep surveying of alternative splicing complexity in the human transcriptome by high-throughput sequencing," Nature Genetics, vol. 40, no. 12, pp. 1413-1415, 2008.

[14] M. Sultan, M. H. Schulz, H. Richard et al., "A global view of gene activity and alternative splicing by deep sequencing of the human transcriptome," Science, vol. 321, no. 5891, pp. 956-960, 2008 .

[15] J. Merkin, C. Russell, P. Chen, and C. B. Burge, "Evolutionary dynamics of gene and isoform regulation in Mammalian tissues," Science, vol. 338, no. 6114, pp. 1593-1599, 2012.

[16] N. L. Barbosa-Morais, M. Irimia, Q. Pan et al., "The evolutionary landscape of alternative splicing in vertebrate species," Science, vol. 338, no. 6114, pp. 1587-1593, 2012. 
[17] F. Heyd and K. W. Lynch, "Degrade, move, regroup: signaling control of splicing proteins," Trends in Biochemical Sciences, vol. 36, no. 8, pp. 397-404, 2011.

[18] A. Kalsotra and T. A. Cooper, "Functional consequences of developmentally regulated alternative splicing," Nature Reviews Genetics, vol. 12, no. 10, pp. 715-729, 2011.

[19] T. A. Cooper, L. Wan, and G. Dreyfuss, "RNA and disease," Cell, vol. 136, no. 4, pp. 777-793, 2009.

[20] R. K. Singh and T. A. Cooper, "Pre-mRNA splicing in disease and therapeutics," Trends in Molecular Medicine, vol. 18, no. 8, pp. 472-482, 2012.

[21] H. Feng, Z. Qin, and X. Zhang, "Opportunities and methods for studying alternative splicing in cancer with RNA-Seq," Cancer Letters, vol. 340, no. 2, pp. 179-191, 2013.

[22] J. Eswaran, A. Horvath, S. Godbole et al., "RNA sequencing of cancer reveals novel splicing alterations," Scientific Reports, vol. 3, article 1689, 2013.

[23] C. J. David and J. L. Manley, "Alternative pre-mRNA splicing regulation in cancer: pathways and programs unhinged," Genes \& Development, vol. 24, no. 21, pp. 2343-2364, 2010.

[24] J. Zhang and J. L. Manley, "Misregulation of pre-mRNA alternative splicing in cancer," Cancer Discovery, vol. 3, no. 11, pp. 1228-1237, 2013.

[25] G. Biamonti, M. Catillo, D. Pignataro, A. Montecucco, and C. Ghigna, "The alternative splicing side of cancer," Seminars in Cell \& Developmental Biology, vol. 32, pp. 30-36, 2014.

[26] D. Hanahan and R. A. Weinberg, "Hallmarks of cancer: the next generation," Cell, vol. 144, no. 5, pp. 646-674, 2011.

[27] C. M. Misquitta-Ali, E. Cheng, D. O’Hanlon et al., "Global profiling and molecular characterization of alternative splicing events misregulated in lung cancer," Molecular and Cellular Biology, vol. 31, no. 1, pp. 138-150, 2011.

[28] J. P. Venables, R. Klinck, A. Bramard et al., "Identification of alternative splicing markers for breast cancer," Cancer Research, vol. 68, no. 22, pp. 9525-9531, 2008.

[29] A. Shilo, V. B. Hur, P. Denichenko et al., "Splicing factor hnRNP A2 activates the Ras-MAPK-ERK pathway by controlling A-Raf splicing in hepatocellular carcinoma development," $R N A$, vol. 20, no. 4, pp. 505-515, 2014.

[30] P. I. Poulikakos, Y. Persaud, M. Janakiraman et al., "RAF inhibitor resistance is mediated by dimerization of aberrantly spliced BRAF(V600E)," Nature, vol. 480, no. 7377, pp. 387-390, 2011.

[31] H. Wang, M. Zhou, B. Shi et al., "Identification of an exon 4-deletion variant of epidermal growth factor receptor with increased metastasis-promoting capacity", Neoplasia, vol. 13, no. 5, pp. 461-471, 2011.

[32] Z. Zhou, J. Qiu, W. Liu et al., “The Akt-SRPK-SR axis constitutes a major pathway in transducing EGF signaling to regulate alternative splicing in the nucleus," Molecular Cell, vol. 47, no. 3, pp. 422-433, 2012.

[33] J.-H. Ding, X.-Y. Zhong, J. C. Hagopian et al., "Regulated cellular partitioning of SR protein-specific kinases in mammalian cells," Molecular Biology of the Cell, vol. 17, no. 2, pp. 876-885, 2006.

[34] S.-W. Jang, X. Liu, H. Fu et al., "Interaction of Akt-phosphorylated SRPK2 with 14-3-3 mediates cell cycle and cell death in neurons," The Journal of Biological Chemistry, vol. 284, no. 36, pp. 24512-24525, 2009.

[35] C. Cheng, M. B. Yaffe, and P. A. Sharp, "A positive feedback loop couples Ras activation and CD44 alternative splicing," Genes and Development, vol. 20, no. 13, pp. 1715-1720, 2006.
[36] H. Ponta, L. Sherman, and P. A. Herrlich, "CD44: from adhesion molecules to signalling regulators," Nature Reviews Molecular Cell Biology, vol. 4, no. 1, pp. 33-45, 2003.

[37] C. Cheng and P. A. Sharp, "Regulation of CD44 alternative splicing by SRm160 and its potential role in tumor cell invasion," Molecular and Cellular Biology, vol. 26, no. 1, pp. 362-370, 2006.

[38] N. Matter, P. Herrlich, and H. König, "Signal-dependent regulation of splicing via phosphorylation of Sam68," Nature, vol. 420, no. 6916, pp. 691-695, 2002.

[39] K. E. Knudsen, "The cyclin D1b splice variant: an old oncogene learns new tricks," Cell Division, vol. 1, article 15, 2006.

[40] K. E. Knudsen, J. Alan Diehl, C. A. Haiman, and E. S. Knudsen, "Cyclin D1: polymorphism, aberrant splicing and cancer risk," Oncogene, vol. 25, no. 11, pp. 1620-1628, 2006.

[41] C. J. Burd, C. E. Petre, L. M. Morey et al., "Cyclin D1b variant influences prostate cancer growth through aberrant androgen receptor regulation," Proceedings of the National Academy of Sciences of the United States of America, vol. 103, no. 7, pp. 21902195, 2006.

[42] P. Bielli, R. Busà, M. P. Paronetto, and C. Sette, "The RNAbinding protein Sam68 is a multifunctional player in human cancer," Endocrine-Related Cancer, vol. 18, no. 4, pp. R91-R102, 2011.

[43] S. Das and A. R. Krainer, "Emerging functions of SRSF1, splicing factor and oncoprotein, in RNA metabolism and cancer," Molecular Cancer Research, vol. 12, no. 9, pp. 1195-1204, 2014.

[44] N. A. Olshavsky, C. E. S. Comstock, M. J. Schiewer et al., "Identification of ASF/SF2 as a critical, allele-specific effector of the cyclin D1b oncogene," Cancer Research, vol. 70, no. 10, pp. 3975-3984, 2010.

[45] M. P. Paronetto, M. Cappellari, R. Busà et al., "Alternative splicing of the cyclin D1 proto-oncogene is regulated by the RNA-binding protein Sam68," Cancer Research, vol. 70, no. 1, pp. 229-239, 2010.

[46] H. Gerhardt, "VEGF and endothelial guidance in angiogenic sprouting," Organogenesis, vol. 4, no. 4, pp. 241-246, 2008.

[47] P. M. Biselli-Chicote, A. R. C. P. Oliveira, E. C. Pavarino, and E. M. Goloni-Bertollo, "VEGF gene alternative splicing: pro- and anti-angiogenic isoforms in cancer," Journal of Cancer Research and Clinical Oncology, vol. 138, no. 3, pp. 363-370, 2012.

[48] K. A. Houck, N. Ferrara, J. Winer, G. Cachianes, B. Li, and D. W. Leung, "The vascular endothelial growth factor family: identification of a fourth molecular species and characterization of alternative splicing of RNA," Molecular Endocrinology, vol. 5, no. 12, pp. 1806-1814, 1991.

[49] D. G. Nowak, J. Woolard, E. M. Amin et al., "Expression of pro- and anti-angiogenic isoforms of VEGF is differentially regulated by splicing and growth factors," Journal of Cell Science, vol. 121, no. 20, pp. 3487-3495, 2008.

[50] A. Mavrou, K. Brakspear, M. Hamdollah-Zadeh et al., "Serinearginine protein kinase 1 (SRPK1) inhibition as a potential novel targeted therapeutic strategy in prostate cancer," Oncogene, 2014.

[51] D. G. Nowak, E. M. Amin, E. S. Rennel et al., "Regulation of vascular endothelial growth factor (VEGF) splicing from pro-angiogenic to anti-angiogenic isoforms: a novel therapeutic strategy for angiogenesis," The Journal of Biological Chemistry, vol. 285, no. 8, pp. 5532-5540, 2010.

[52] E. M. Amin, S. Oltean, J. Hua et al., "WT1 mutants reveal SRPK1 to be a downstream angiogenesis target by altering VEGF splicing," Cancer Cell, vol. 20, no. 6, pp. 768-780, 2011. 
[53] M. A. H. Zadeh, E. M. Amin, C. Hoareau-Aveilla et al., "Alternative splicing of TIA-1 in human colon cancer regulates VEGF isoform expression, angiogenesis, tumour growth and bevacizumab resistance," Molecular Oncology, vol. 9, no. 1, pp. 167-178, 2015.

[54] S. Eswarappa, A. Potdar, W. Koch et al., "Programmed translational readthrough generates antiangiogenic VEGF-Ax," Cell, vol. 157, no. 7, pp. 1605-1618, 2014.

[55] M. Salton, T. C. Voss, and T. Misteli, "Identification by highthroughput imaging of the histone methyltransferase EHMT2 as an epigenetic regulator of VEGFA alternative splicing," Nucleic Acids Research, vol. 42, no. 22, pp. 13662-13673, 2014.

[56] S. Valastyan and R. A. Weinberg, "Tumor metastasis: molecular insights and evolving paradigms," Cell, vol. 147, no. 2, pp. 275292, 2011.

[57] B. de Craene and G. Berx, "Regulatory networks defining EMT during cancer initiation and progression," Nature Reviews Cancer, vol. 13, no. 2, pp. 97-110, 2013.

[58] I. M. Shapiro, A. W. Cheng, N. C. Flytzanis et al., "An EMTdriven alternative splicing program occurs in human breast cancer and modulates cellular phenotype," PLoS Genetics, vol. 7, no. 8, Article ID e1002218, 2011.

[59] C. C. Warzecha and R. P. Carstens, "Complex changes in alternative pre-mRNA splicing play a central role in the epithelialto-mesenchymal transition (EMT)," Seminars in Cancer Biology, vol. 22, no. 5-6, pp. 417-427, 2012.

[60] R. L. Brown, L. M. Reinke, M. S. Damerow et al., "CD44 splice isoform switching in human and mouse epithelium is essential for epithelial-mesenchymal transition and breast cancer progression," Journal of Clinical Investigation, vol. 121, no. 3, pp. 1064-1074, 2011.

[61] F. di Modugno, P. Iapicca, A. Boudreau et al., "Splicing program of human MENA produces a previously undescribed isoform associated with invasive, mesenchymal-like breast tumors," Proceedings of the National Academy of Sciences of the United States of America, vol. 109, no. 47, pp. 19280-19285, 2012.

[62] K. Horiguchi, K. Sakamoto, D. Koinuma et al., “TGF-beta drives epithelial-mesenchymal transition through deltaEF1-mediated downregulation of ESRP," Oncogene, vol. 31, no. 26, pp. 31903201, 2012.

[63] L. M. Reinke, Y. Xu, and C. Cheng, "Snail represses the splicing regulator epithelial splicing regulatory protein 1 to promote epithelial-mesenchymal transition," Journal of Biological Chemistry, vol. 287, no. 43, pp. 36435-36442, 2012.

[64] C. C. Warzecha, P. Jiang, K. Amirikian et al., "An ESRP-regulated splicing programme is abrogated during the epithelialmesenchymal transition," The EMBO Journal, vol. 29, no. 19, pp. 3286-3300, 2010.

[65] F. Pelisch, D. Khauv, G. Risso et al., "Involvement of hnRNP A1 in the matrix metalloprotease-3-dependent regulation of Racl pre-mRNA splicing," Journal of Cellular Biochemistry, vol. 113, no. 7, pp. 2319-2329, 2012.

[66] D. C. Radisky, D. D. Levy, L. E. Littlepage et al., "Raclb and reactive oxygen species mediate MMP-3-induced EMT and genomic instability," Nature, vol. 436, no. 7047, pp. 123-127, 2005.

[67] V. Goncalves, A. Henriques, J. Pereira et al., "Phosphorylation of SRSF1 by SRPK1 regulates alternative splicing of tumor-related Raclb in colorectal cells," RNA, vol. 20, no. 4, pp. 474-482, 2014.

[68] C. Ghigna, S. Giordano, H. Shen et al., "Cell motility is controlled by SF2/ASF through alternative splicing of the Ron protooncogene," Molecular Cell, vol. 20, no. 6, pp. 881-890, 2005.
[69] C. Collesi, M. M. Santoro, G. Gaudino, and P. M. Comoglio, "A splicing variant of the RON transcript induces constitutive tyrosine kinase activity and an invasive phenotype," Molecular and Cellular Biology, vol. 16, no. 10, pp. 5518-5526, 1996.

[70] C. Valacca, S. Bonomi, E. Buratti et al., "Sam68 regulates EMT through alternative splicing-activated nonsense-mediated mRNA decay of the SF2/ASF proto-oncogene," Journal of Cell Biology, vol. 191, no. 1, pp. 87-99, 2010.

[71] I. Cascino, G. Fiucci, G. Papoff, and G. Ruberti, "Three functional soluble forms of the human apoptosis-inducing Fas molecule are produced by alternative splicing," The Journal of Immunology, vol. 154, no. 6, pp. 2706-2713, 1995.

[72] J. Cheng, T. Zhou, C. Liu et al., "Protection from Fas-mediated apoptosis by a soluble form of the Fas molecule," Science, vol. 263, no. 5154, pp. 1759-1762, 1994.

[73] J. M. Izquierdo, N. Majós, S. Bonnal et al., "Regulation of fas alternative splicing by antagonistic effects of TIA- 1 and PTB on exon definition," Molecular Cell, vol. 19, no. 4, pp. 475-484, 2005.

[74] M. P. Paronetto, I. Bernardis, E. Volpe et al., "Regulation of FAS exon definition and apoptosis by the Ewing sarcoma protein," Cell Reports, vol. 7, no. 4, pp. 1211-1226, 2014.

[75] S. Bonnal, C. Martínez, P. Förch, A. Bachi, M. Wilm, and J. Valcárcel, "RBM5/Luca-15/H37 regulates Fas alternative splice site pairing after exon definition," Molecular Cell, vol. 32, no. 1, pp. 81-95, 2008.

[76] J. M. Izquierdo, "Hu antigen R (HuR) functions as an alternative pre-mRNA splicing regulator of Fas apoptosis-promoting receptor on exon definition," The Journal of Biological Chemistry, vol. 283, no. 27, pp. 19077-19084, 2008.

[77] J. M. Izquierdo, "Heterogeneous ribonucleoprotein C displays a repressor activity mediated by T-cell intracellular antigen-1related/like protein to modulate Fas exon 6 splicing through a mechanism involving $\mathrm{Hu}$ antigen R," Nucleic Acids Research, vol. 38, no. 22, pp. 8001-8014, 2010.

[78] D.-W. Seol and T. R. Billiar, "A caspase-9 variant missing the catalytic site is an endogenous inhibitor of apoptosis," Journal of Biological Chemistry, vol. 274, no. 4, pp. 2072-2076, 1999.

[79] S. M. Srinivasula, M. Ahmad, Y. Guo et al., "Identification of an endogenous dominant-negative short isoform of caspase-9 that can regulate apoptosis," Cancer Research, vol. 59, no. 5, pp. 999-1002, 1999.

[80] J. C. Shultz, R. W. Goehe, D. S. Wijesinghe et al., "Alternative splicing of caspase 9 is modulated by the phosphoinositide 3kinase/Akt pathway via phosphorylation of SRp30a," Cancer Research, vol. 70, no. 22, pp. 9185-9196, 2010.

[81] R. W. Goehe, J. C. Shultz, C. Murudkar et al., "hnRNP L regulates the tumorigenic capacity of lung cancer xenografts in mice via caspase- 9 pre-mRNA processing," The Journal of Clinical Investigation, vol. 120, no. 11, pp. 3923-3939, 2010.

[82] L. H. Boise, M. González-García, C. E. Postema et al., "bcl-x, a bcl-2-related gene that functions as a dominant regulator of apoptotic cell death," Cell, vol. 74, no. 4, pp. 597-608, 1993.

[83] D. Garneau, T. Revil, J.-F. Fisette, and B. Chabot, "Heterogeneous nuclear ribonucleoprotein $\mathrm{F} / \mathrm{H}$ proteins modulate the alternative splicing of the apoptotic mediator Bcl-x," The Journal of Biological Chemistry, vol. 280, no. 24, pp. 22641-22650, 2005.

[84] P. Bielli, M. Bordi, V. D. Biasio, and C. Sette, "Regulation of BCL$\mathrm{X}$ splicing reveals a role for the polypyrimidine tract binding protein (PTBP1/hnRNP I) in alternative $5^{\prime}$ splice site selection," Nucleic Acids Research, vol. 42, no. 19, pp. 12070-12081, 2014. 
[85] M. P. Paronetto, T. Achsel, A. Massiello, C. E. Chalfant, and C. Sette, "The RNA-binding protein Sam68 modulates the alternative splicing of Bcl-x," Journal of Cell Biology, vol. 176, no. 7, pp. 929-939, 2007.

[86] A. Zhou, A. C. Ou, A. Cho, E. J. Benz Jr., and S.-C. Huang, "Novel splicing factor RBM25 modulates Bcl-x Pre-mRNA 5' splice site selection," Molecular and Cellular Biology, vol. 28, no. 19, pp. 5924-5936, 2008.

[87] S. Pedrotti, R. Busà, C. Compagnucci, and C. Sette, "The RNA recognition motif protein RBM11 is a novel tissue-specific splicing regulator," Nucleic Acids Research, vol. 40, no. 3, pp. 1021-1032, 2012.

[88] A. Massiello, J. R. Roesser, and C. E. Chalfant, "SAP155 Binds to ceramide-responsive RNA cis-element 1 and regulates the alternative $5^{\prime}$ splice site selection of Bcl-x pre-mRNA," The FASEB Journal, vol. 20, no. 10, pp. 1680-1682, 2006.

[89] P. Cloutier, J. Toutant, L. Shkreta, S. Goekjian, T. Revil, and B. Chabot, "Antagonistic effects of the SRp30c protein and cryptic 5/ splice sites on the alternative splicing of the apoptotic regulator Bcl-x," The Journal of Biological Chemistry, vol. 283, no. 31, pp. 21315-21324, 2008.

[90] T. Revil, J. Pelletier, J. Toutant, A. Cloutier, and B. Chabot, "Heterogeneous nuclear ribonucleoprotein $\mathrm{K}$ represses the production of pro-apoptotic Bcl-xS splice isoform," The Journal of Biological Chemistry, vol. 284, no. 32, pp. 21458-21467, 2009.

[91] M. J. Moore, Q. Wang, C. J. Kennedy, and P. A. Silver, "An alternative splicing network links cell-cycle control to apoptosis," Cell, vol. 142, no. 4, pp. 625-636, 2010.

[92] J. A. Bauman, S.-D. Li, A. Yang, L. Huang, and R. Kole, "Antitumor activity of splice-switching oligonucleotides," Nucleic Acids Research, vol. 38, no. 22, pp. 8348-8356, 2010.

[93] D. R. Mercatante, J. L. Mohler, and R. Kole, "Cellular response to an antisense-mediated shift of Bcl-x pre-mRNA splicing and antineoplastic agents," The Journal of Biological Chemistry, vol. 277, no. 51, pp. 49374-49382, 2002.

[94] O. Warburg, "On the origin of cancer cells," Science, vol. 123, no. 3191, pp. 309-314, 1956.

[95] O. Warburg, "On respiratory impairment in cancer cells," Science, vol. 124, no. 3215, pp. 269-270, 1956.

[96] M. G. V. Heiden, L. C. Cantley, and C. B. Thompson, "Understanding the warburg effect: the metabolic requirements of cell proliferation," Science, vol. 324, no. 5930, pp. 1029-1033, 2009.

[97] H. R. Christofk, M. G. Vander Heiden, M. H. Harris et al., “The M2 splice isoform of pyruvate kinase is important for cancer metabolism and tumour growth," Nature, vol. 452, no. 7184, pp. 230-233, 2008.

[98] W. Yang, Y. Xia, Y. Cao et al., "EGFR-induced and PKCe monoubiquitylation-dependent NF- $\kappa \mathrm{B}$ activation upregulates PKM2 expression and promotes tumorigenesis," Molecular Cell, vol. 48, no. 5, pp. 771-784, 2012.

[99] W. Yang, Y. Xia, D. Hawke et al., "PKM2 phosphorylates histone $\mathrm{H} 3$ and promotes gene transcription and tumorigenesis," Cell, vol. 150, no. 4, pp. 685-696, 2012.

[100] C. V. Clower, D. Chatterjee, Z. Wang, L. C. Cantley, M. G. V. Heidena, and A. R. Krainer, "The alternative splicing repressors hnRNP A1/A2 and PTB influence pyruvate kinase isoform expression and cell metabolism," Proceedings of the National Academy of Sciences of the United States of America, vol. 107, no. 5, pp. 1894-1899, 2010.

[101] C. J. David, M. Chen, M. Assanah, P. Canoll, and J. L. Manley, "HnRNP proteins controlled by c-Myc deregulate pyruvate kinase mRNA splicing in cancer," Nature, vol. 463, no. 7279, pp. 364-368, 2010.

[102] V. Fodale, M. Pierobon, L. Liotta, and E. Petricoin, "Mechanism of cell adaptation: when and how do cancer cells develop chemoresistance?” Cancer Journal, vol. 17, no. 2, pp. 89-95, 2011.

[103] Y. Wang, J. L. Dean, E. K. A. Millar et al., "Cyclin D1b is aberrantly regulated in response to therapeutic challenge and promotes resistance to estrogen antagonists," Cancer Research, vol. 68, no. 14, pp. 5628-5638, 2008.

[104] J. W. Lee, Y. H. Soung, S. H. Seo et al., "Somatic mutations of ERBB2 kinase domain in gastric, colorectal, and breast carcinomas," Clinical Cancer Research, vol. 12, no. 1, pp. 57-61, 2006.

[105] P. Stephens, C. Hunter, G. Bignell et al., "Lung cancer: intragenic ERBB2 kinase mutations in tumours," Nature, vol. 431, no. 7008, pp. 525-526, 2004.

[106] C. I. Zito, D. Riches, J. Kolmakova, J. Simons, M. Egholm, and D. F. Stern, "Direct resequencing of the complete ERBB2 coding sequence reveals an absence of activating mutations in ERBB2 amplified breast cancer," Genes Chromosomes and Cancer, vol. 47, no. 7, pp. 633-638, 2008.

[107] F. Castiglioni, E. Tagliabue, M. Campiglio, S. M. Pupa, A. Balsari, and S. Ménard, "Role of exon-16-deleted HER2 in breast carcinomas," Endocrine-Related Cancer, vol. 13, no. 1, pp. 221232, 2006.

[108] K. Y. Kwong and M. C. Hung, "A novel splice variant of HER2 with increased transformation activity," Molecular Carcinogenesis, vol. 23, no. 2, pp. 62-68, 1998.

[109] P. M. Siegel, E. D. Ryan, R. D. Cardiff, and W. J. Muller, "Elevated expression of activated forms of Neu/ErbB-2 and ErbB-3 are involved in the induction of mammary tumors in transgenic mice: implications for human breast cancer," The EMBO Journal, vol. 18, no. 8, pp. 2149-2164, 1999.

[110] D. Mitra, M. J. Brumlik, S. U. Okamgba et al., "An oncogenic isoform of HER2 associated with locally disseminated breast cancer and trastuzumab resistance," Molecular Cancer Therapeutics, vol. 8, no. 8, pp. 2152-2162, 2009.

[111] W. L. Perry III, R. L. Shepard, J. Sampath et al., "Human splicing factor SPF45 (RBM17) confers broad multidrug resistance to anticancer drugs when overexpressed-a phenotype partially reversed by selective estrogen receptor modulators," Cancer Research, vol. 65, no. 15, pp. 6593-6600, 2005.

[112] J. Sampath, P. R. Long, R. L. Shepard et al., "Human SPF45, a splicing factor, has limited expression in normal tissues, is overexpressed in many tumors, and can confer a multidrugresistant phenotype to cells," The American Journal of Pathology, vol. 163, no. 5, pp. 1781-1790, 2003.

[113] A. M. Al-Ayoubi, H. Zheng, Y. Liu, T. Bai, and S. T. Eblen, "Mitogen-activated protein kinase phosphorylation of splicing factor 45 (SPF45) regulates SPF45 alternative splicing site utilization, proliferation, and cell adhesion," Molecular and Cellular Biology, vol. 32, no. 14, pp. 2880-2893, 2012.

[114] L. Corsini, S. Bonnal, J. Basquin et al., "U2AF-homology motif interactions are required for alternative splicing regulation by SPF45," Nature Structural and Molecular Biology, vol. 14, no. 7, pp. 620-629, 2007.

[115] Y. Liu, L. Conaway, J. Rutherford Bethard et al., "Phosphorylation of the alternative mRNA splicing factor 45 (SPF45) by Clk1 regulates its splice site utilization, cell migration and invasion," Nucleic Acids Research, vol. 41, no. 9, pp. 4949-4962, 2013.

[116] S. M. Dehm, L. J. Schmidt, H. V. Heemers, R. L. Vessella, and D. J. Tindall, "Splicing of a novel androgen receptor exon generates 
a constitutively active androgen receptor that mediates prostate cancer therapy resistance," Cancer Research, vol. 68, no. 13, pp. 5469-5477, 2008.

[117] T. Sugiyama, Y. Nishio, T. Kishimoto, and S. Akira, "Identification of alternative splicing form of stat2," FEBS Letters, vol. 381, no. 3, pp. 191-194, 1996.

[118] N. Zhang and Y.-W. He, "An essential role for c-FLIP in the efficient development of mature T lymphocytes," The Journal of Experimental Medicine, vol. 202, no. 3, pp. 395-404, 2005.

[119] R. Busà, R. Geremia, and C. Sette, "Genotoxic stress causes the accumulation of the splicing regulator Sam68 in nuclear foci of transcriptionally active chromatin," Nucleic Acids Research, vol. 38, no. 9, pp. 3005-3018, 2010.

[120] R. Busà, M. P. Paronetto, D. Farini et al., "The RNA-binding protein Sam68 contributes to proliferation and survival of human prostate cancer cells," Oncogene, vol. 26, no. 30, pp. 4372-4382, 2007.

[121] M. P. Paronetto, B. Miñana, and J. Valcárcel, “The Ewing sarcoma protein regulates DNA damage-induced alternative splicing," Molecular Cell, vol. 43, no. 3, pp. 353-368, 2011.

[122] L. Adesso, S. Calabretta, F. Barbagallo et al., "Gemcitabine triggers a pro-survival response in pancreatic cancer cells through activation of the MNK2/eIF4E pathway," Oncogene, vol. 32, no. 23, pp. 2848-2857, 2013.

[123] A. Maimon, M. Mogilevsky, A. Shilo et al., "Mnk2 alternative splicing modulates the p38-MAPK pathway and impacts Rasinduced transformation," Cell Reports, vol. 7, no. 2, pp. 501-513, 2014.

[124] K. Lenos, A. M. Grawenda, K. Lodder et al., "Alternate splicing of the p53 inhibitor HDMX offers a superior prognostic biomarker than p53 mutation in human cancer," Cancer Research, vol. 72, no. 16, pp. 4074-4084, 2012.

[125] J. C. Shultz, R. W. Goehe, C. S. Murudkar et al., "SRSF1 regulates the alternative splicing of caspase 9 via a novel intronic splicing enhancer affecting the chemotherapeutic sensitivity of nonsmall cell lung cancer cells," Molecular Cancer Research, vol. 9, no. 7, pp. 889-900, 2011.

[126] Z. Wang, H. Y. Jeon, F. Rigo, C. F. Bennett, and A. R. Krainer, "Manipulation of PK-M mutually exclusive alternative splicing by antisense oligonucleotides," Open Biology, vol. 2, no. 10, article 120133, 2012.

[127] F. Rigo, S. J. Chun, D. A. Norris et al., "Pharmacology of a central nervous system delivered 2 '-O-methoxyethyl-modified survival of motor neuron splicing oligonucleotide in mice and nonhuman primates," Journal of Pharmacology and Experimental Therapeutics, vol. 350, no. 1, pp. 46-55, 2014. 

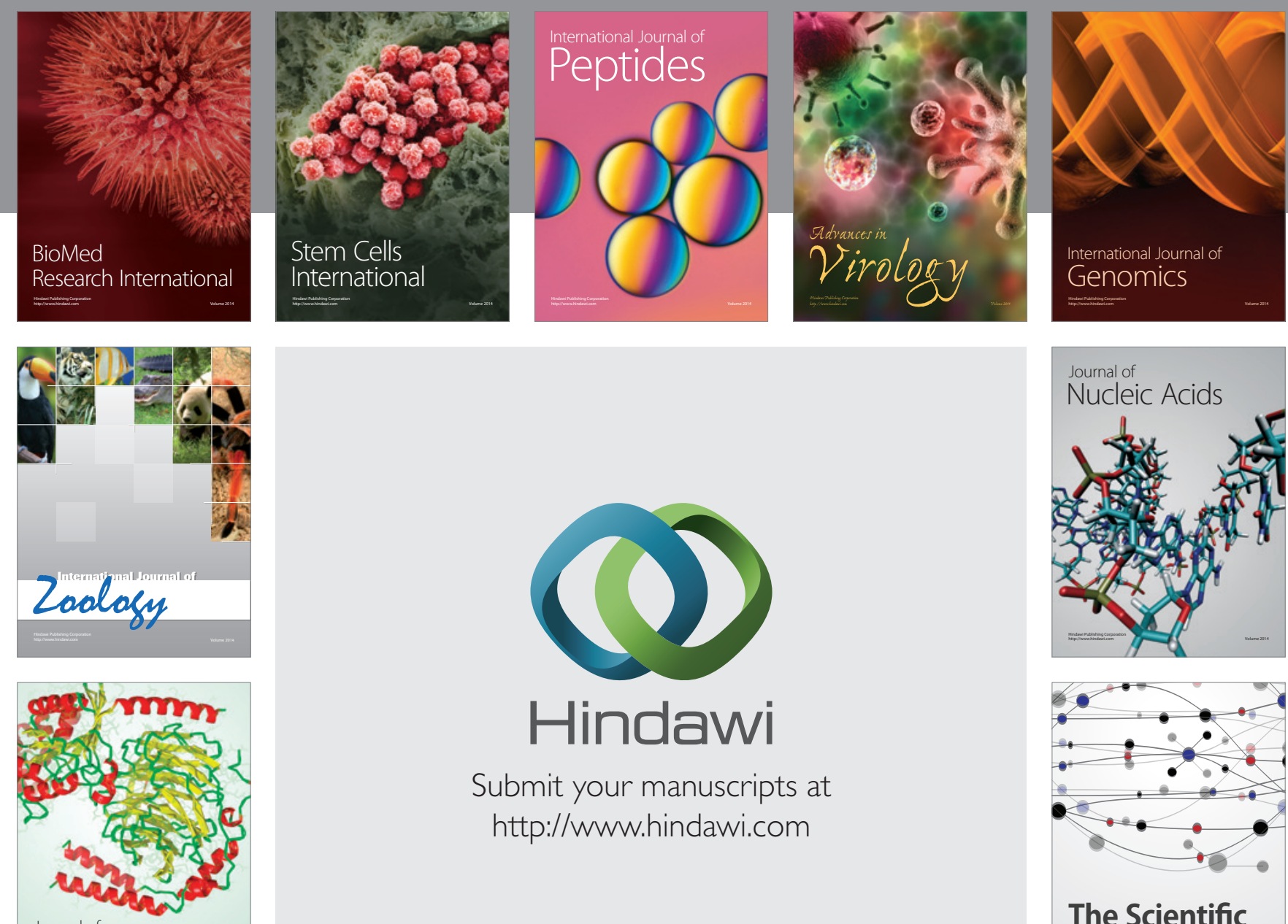

Submit your manuscripts at

http://www.hindawi.com

Journal of
Signal Transduction
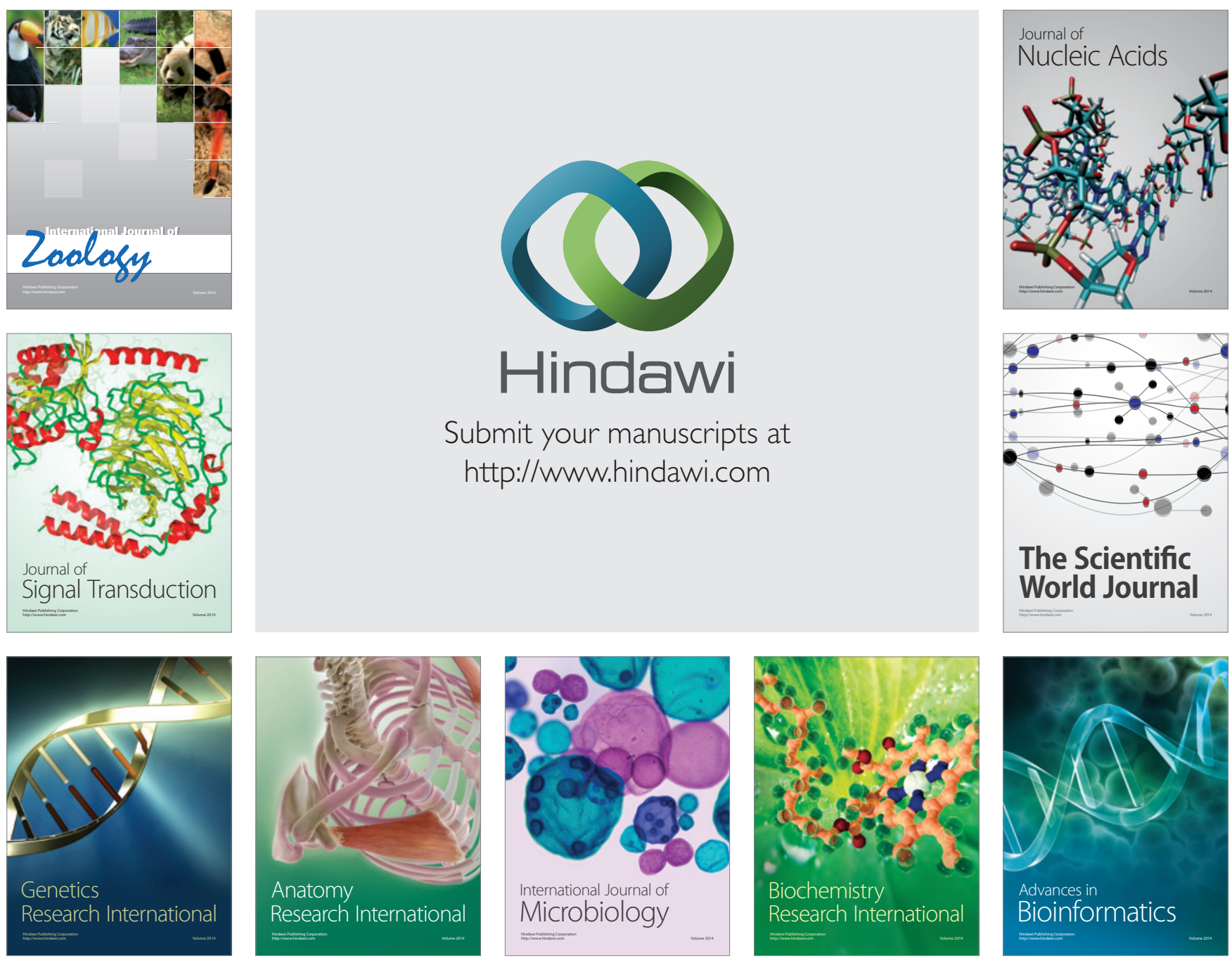

The Scientific World Journal
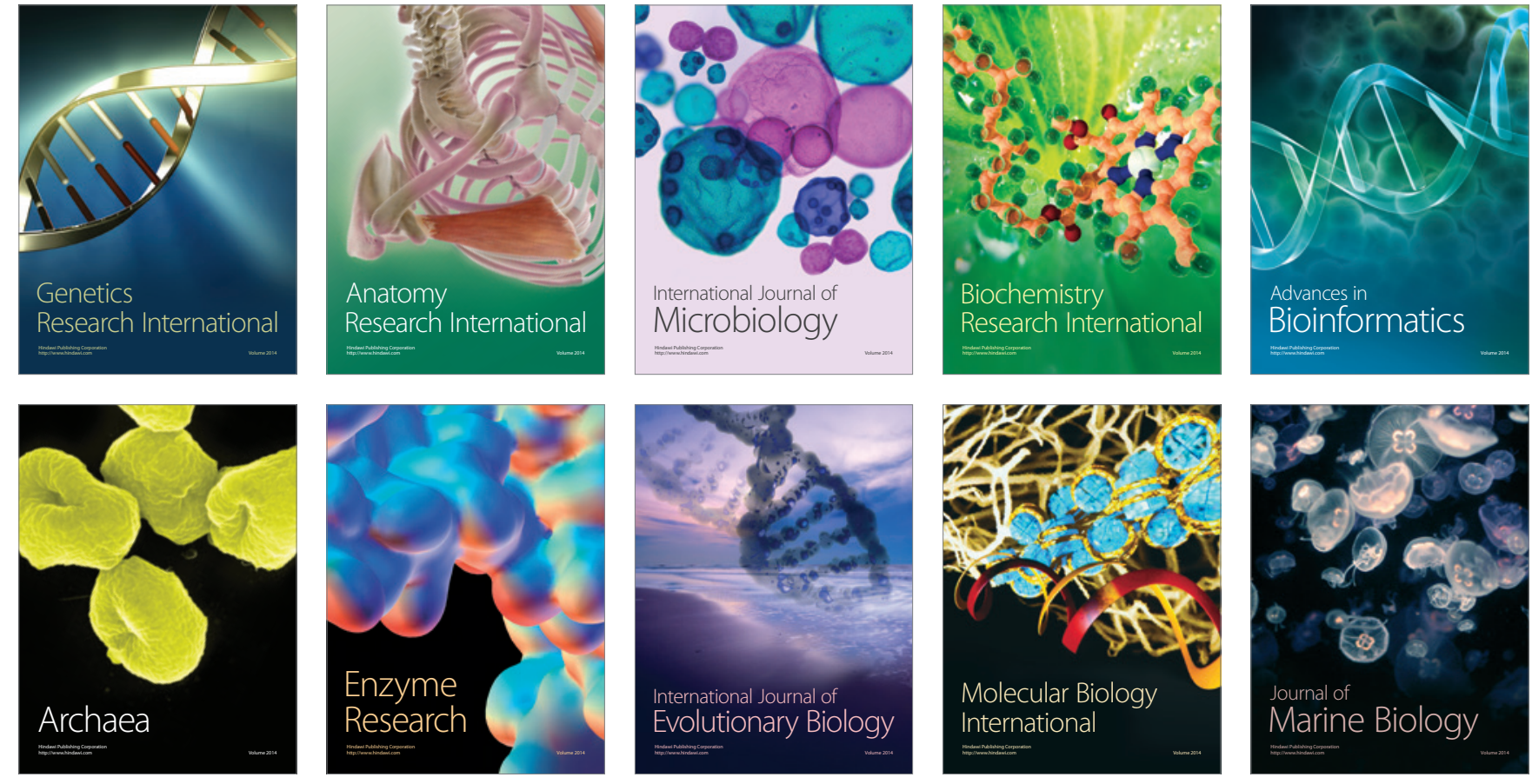